work, with a mass of useful information compressed into a small space. Dr. J. Gross deals with the thyroid hormones and includes an account of the recent discoveries which he and Mrs. R. Pitt-Rivers have made on the various iodine compounds in the thyroid gland and in the blood. Much of this important work would have been impossible without the use of paper chromatography in combination with tracer technique using radioactive iodine, which permitted analyses to be made on an ultra-micro scale. This article, and the one by Drs. C. E. Dent and J. M. Walshe on amino-acid metabolism, are of direct medical appeal.

Some of the articles contain a good deal of hitherto unpublished work, and each includes a bibliography which greatly adds to the value of the publication. Incidentally, references to Nature, which is being regularly used as a forum for the recording and exchange of new chromatographic procedures, appear frequently in these bibliographies. It seems a pity that all the illustrations in a volume entitled "Chromatography" should be produced in black-andwhite. Some private journals, notably Endeavour, have shown what æesthetically pleasing results can be achieved by the use of colour in illustrating chromatographic techniques, even if, from a purely scientific point of view, the gain does not justify the large extra cost. No worker who is interested in chromatography, whether beginner or expert, can fail to benefit from reading this admirable publication. It is likely to be used on the laboratory bench as well as in the library, and it deserves to be protected from accidental 'paper chromatography' by being given a permanent, binding.

D. C. HARRISON

\section{THE EXPANDING UNIVERSE}

A SLIGHTLY modified version of a talk given by G. J. Whitrow in February 1953 in the B.B.C. Third Programme has now been published as a paper under the title of "The Orthodox Theory of the Expanding Universe" in the October issue of Occasional Notes of the Royal Astronomical Society $(17,3 ;$ 1954). A fairly large portion of the talk is concerned with a work by Paul Couderc, a French astronomer, which appeared in a French edition in 1950 and was translated into English by J. B. Sidgwick under the title "The Expansion of the Universe" (1952). The first part of the talk gives a very useful résumé of the developments in our knowledge of the universe during the past thirty years, in which period it was accepted that the universe extends far beyond the limits of our galaxy, and also that the red shift strongly suggested the recession of the extra-galactic nebula, all of which were moving away from one another. The talk then considers other interpretations that have been given to the displacement towards the red end of the spectrum, dealing in particular with various matters connected with Couderc's book.

This book is recognized as a very useful contribution to cosmology in so far as it provides us with a simplified account of the mathematical theory of the expanding universe ; but in recent years some British cosmologists have advocated a new theory based on the hypothesis of the continuous creation of matter. Differences of view arise regarding the form in which this new matter emerges. C. Pascual Jordan advocates the formation of super-giant stars; but other cosmologists who challenge this view have put forward the conception of new matter appearing in the form of hydrogen molecules or even neutrons, and the density of the universe remains constant, the decrease in density which would result from the recession of the galaxies being exactly balanced by the continuous creation of matter. As might be expected, these theories have been subjected to severe criticisms; nevertheless, they are able to claim one strong argument in their favour-that they allow more time for stellar evolution than the older orthodox view which led to a conception of the earth being older than the galaxies. This argument is no longer valid since Baade's announcement about the wrong 'calibration' of the distances of the Cepheid variables (the investigations of Thackeray and Wesselink on the distance of the Magellanic Clouds have corroborated Baade's conclusions); now it seems that it must have been about four thousand million years ago since the universe started expanding, and in addition, the distances of the extra-galactic nebulæ must now be taken as twice those previously estimated.

Whitrow concludes with an expression of disagreement with Couderc's acceptance of the two fundamental hypotheses at the basis of the 'orthodox' theory of the expanding universe; these are that the red shifts are due to the Doppler effect associated with recessional motion, and also that the laws of Einstein's general theory of relativity, either with or without the idea of cosmical repulsion, govern the structure of the universe as a whole. He thinks that neither of these is on such firm foundations as Coudere believes; but in spite of this disagreement he speaks highly of the latter's book which, on p. 213, seems to express a little doubt on the inadequacy of some of his own arguments. "Everything possible has been extracted from existing observations; until the appearance of new data we would be wise to give our imaginations a rest." To this remark Whitrow makes the very relevant reply : ". . . if such advice had been accepted by Copernicus he would never have ventured beyond the Ptolemaic theory".

\section{RECENT MOLLUSCA OF CANADA}

$\mathrm{T}$ HE great extent of Canada has resulted, and will probably long result, in its molluscan fauna being studied in four separate areas: the Eastern Provinces, British Columbia, the Arctic region based on Hudson Bay, and the Prairie Provinces. In the first three, marine, freshwater and land forms are involved, but in the fourth only freshwater and land. Partly for this reason and also because many of the species were first described from other countries, the literature involved is extensive and scattered.

A very useful service in rendering this literature and its results available for the whole of Canada has been performed by the publication of Bulletin No. 129 of the National Museum of Canada, entitled "Cata. logue of the Recent Mollusca of Canada", by Dr. Aurèle La Rocque (Biological Series No. 44. Pp. ix +406. Ottawa: Queon's Printer, 1953; 2.50 dollars). It fills a very obvious gap, for it provides a list of the accepted species and forms that have so far been recorded, numbering about 2,250. The author has wisely included forms that have actually 
only been described from adjacent seas and land areas, where it may reasonably be inferred that more extensive collecting will lead to their being found within the strict territorial limits. Under each form are given the original reference and, where known, the type locality, references to papers containing further data, and the known range. A complete synonymy is not given for any species; but it can be obtained from the references. In some instances one or, more rarely, two synonyms in use in previous Canadian records are provided. Future collecting will no doubt add to the list considerably; but it will also be liable to reduce it when the method adopted by Hubendick for Lymnaea peregra is applied to some of the freshwater forms. The number of species of some genera, for example, Odostomia from the Pacific coast, that are only known from the one record from the type locality, implies that more extensive collecting will show some of them to be members of highly variable rather than separate species.

Two minor points suggest that a more careful check of dates might have been made : for example, on p. 2, the author states that it is unnecessary to give references to the original founding of genera because they are given by Neave (1935), but, as correctly cited in the bibliography, the earliest date of Neave is 1939 ; the date of Mozley's paper on Stagnicola traskii castorensis is given as 1930 in the text, but 1931 in the bibliography. However, on the whole there is nothing but praise for this well-printed and well-presented catalogue, which could only have been prepared so satisfactorily by one like Dr. La Rocque, who combines critical enthusiasm with a long personal acquaintance with his subject.

\section{INFLUENCE OF MONOLAYERS ON THE NATURAL RATE OF EVAPORATION OF WATER}

\section{By W. W. MANSFIELD \\ Division of Industrial Chemistry, Commonwealth Scientific and Industrial Research Organization, Melbourne}

$\mathrm{F}^{\mathrm{o}}$

OR many years it has been known that the presence on water surfaces of monolayers of $\mathbf{a}$ number of long-chain compounds or of layers of appropriate oil mixtures may restrict the evaporation of water 1,2. Apart from this property, a film suitable for reducing natural evaporation from water storages must resist the actions of wind and dust, must be reasonably permanent and capable of ready and economic application, should not restrict markedly the access of air and sunlight to the water, and must not be toxic. In this communication there are summarized a number of results which indicate that monolayers of cetyl alcohol satisfy these requirements.

Action of wind and dust. In this laboratory it has been found that rigid films, such as monolayers of long-chain fatty acids or oil layers containing appropriate additives, are damaged by both wind and dust. As the rigidity of the film also hinders repair, the ability to retard evaporation is lost rapidly.

With more fluid oil films, the inevitable reversion to the system of lenses in equilibrium with a mono. layer is hastened by wind and dust. A monolayer of this type of film does not offer great resistance to the transfer of water vapour, and the restriction provided by the lenses is reduced markedly by settled dust. Accordingly, under natural conditions the efficiency of such systems decays rapidly.

Provided excess crystals of the solid from which it is derived are available, the efficiency of a liquid monolayer, such as that formed by a long-chain alcohol, is unimpaired by the action of wind. It has been observed also that the performance of this system is not diminished by bombardment with dust; in fact, the resistance to transfer of vapour is increased temporarily. Only materials such as powdered talc and carbon, capable of remaining on the surface for some time, cause a decline in efficiency. The occurrence of considerable proportions of these substances in natural dust is not common.

Performance of monolayers. The resistances offered to the transfer of water vapour by a number of monolayers, formed on still surfaces by spreading from the solid, have been determined. The apparatus used resembled that described by Langmuir and Schaefer ${ }^{3}$. It has been found that straight-chain alcohols generate monolayers providing greater retardation than those formed from alcohols containing branched chains or ring skeletons within the molecule. For the straight-chain alcohols, most efficient restriction is obtained with monolayers of cetyl or stearyl alcohol. At room temperature, these two films exhibit very similar behaviour, offering a resistance to vapour transfer about 1,600 times greater than that of a clean water surface, for which an accommodation coefficient of $0.036^{4,5}$ has been assumed. The accommodation coefficient of monolayers does increase with temperature, however; for cetyl alcohol the rise is from $2.2 \times 10^{-5}$ at $20^{\circ} \mathrm{C}$. to $7 \cdot 9 \times 10^{-5}$ at $50^{\circ} \mathrm{C}$.

Out of doors, the laminar layer of air above a water surface provides a resistance which is normally about eight hundred times that of the water surface alone. The presence of $a$ monolayer of cetyl alcohol results in a total resistance which is three times greater ; accordingly, the monolayer should reduce natural evaporation by about 70 per cent. Any reduction in evaporation, however, promotes an increased surface temperature. The consequent compensation, the extent of which varies with the wind velocity, the depth of water and the radiation received by the water, is not excessive for average conditions. A previous conclusion ${ }^{8}$ that this compensating effect prevents significant control of ovaporation is quite incorrect; it was influenced by poor results obtained with imperfectly developed monolayers spread on small and shallow samples of water. The construction of heat balances, somewhat after the manner of Ferguson ${ }^{7}$, predicts a mean reduction of about 45 per cent for normal summer conditions in inland southern Australia.

Diminution of evaporation to this extent admittedly is not spectacular, but may be achieved without any significant decrease in the rate of access of oxygen or other gases to the water, and also without hindering the penetration of light. Severe restriction of evaporation by a film alone should be avoided, because the influx of oxygen may be retarded sufficiently to promote stagnation. Similarly, the combined use of floating reflecting surfaces and a monolayer ${ }^{6}$ inhibits evaporation to a much larger extent than does a monolayer alone, but the entry of sunlight is restricted also.

Attainment of optimum performance. The estimates presented are based on results obtained for a mono- 